\title{
Acceptability and Feasibility of Sexually Transmitted Infection Testing and Treatment among Pregnant Women in Gaborone, Botswana, 2015
}

\author{
Adriane Wynn, ${ }^{1}$ Doreen Ramogola-Masire, ${ }^{2,3}$ \\ Ponatshego Gaolebale, ${ }^{4}$ Neo Moshashane, ${ }^{3}$ Ogechukwu Agatha Offorjebe, ${ }^{5,6}$ \\ Kaitlin Arena, ${ }^{6}$ Jeffrey D. Klausner, ${ }^{7}$ and Chelsea Morroni ${ }^{2,8,9}$ \\ ${ }^{1}$ Department of Health Policy and Management, Fielding School of Public Health, University of California, Los Angeles, \\ 650 Charles Young Dr. S., 31-269 CHS, P.O. Box 951772, Los Angeles, CA 90095-1772, USA \\ ${ }^{2}$ Botswana-UPenn Partnership, 244G UB Main Campus, Gaborone, Botswana \\ ${ }^{3}$ University of Botswana, 4775 Notwane Road, Gaborone, Botswana \\ ${ }^{4}$ Princess Marina Hospital, Phologolo Extension 9, Gaborone, Botswana \\ ${ }^{5}$ Charles R. Drew University of Medicine \& Science, 1731 E. 120th Street, Los Angeles, CA 90059, USA \\ ${ }^{6}$ David Geffen School of Medicine, University of California, Los Angeles, 10833 Le Conte Avenue, Los Angeles, CA 90095, USA \\ ${ }^{7}$ Division of Infectious Diseases, Department of Epidemiology, David Geffen School of Medicine and Fielding School of Public Health, \\ University of California, Los Angeles, 10833 Le Conte Avenue, CHS 13-154a, Los Angeles, CA 90095, USA \\ ${ }^{8}$ EGA Institute for Women's Health and Institute for Global Health, University College London, 74 Huntley Street, \\ London WC1E 6AU, UK \\ ${ }^{9}$ Wits Reproductive Health and HIV Institute, University of Witwatersrand, 58 Klein Street, Johannesburg 2001, South Africa
}

Correspondence should be addressed to Adriane Wynn; adriane.wynn@gmail.com

Received 30 October 2015; Accepted 18 January 2016

Academic Editor: Kasonde Mwinga

Copyright (C) 2016 Adriane Wynn et al. This is an open access article distributed under the Creative Commons Attribution License, which permits unrestricted use, distribution, and reproduction in any medium, provided the original work is properly cited.

\begin{abstract}
Introduction. Chlamydia trachomatis (CT), Neisseria gonorrhoeae (NG), and Trichomonas vaginalis (TV) are curable sexually transmitted infections (STIs) that can cause adverse maternal and birth outcomes. Most countries do not conduct routine testing during antenatal care. We present data on the acceptability and feasibility of testing and treating pregnant women for STIs in an antenatal clinic in Gaborone, Botswana. Materials and Methods. We offered CT, NG, and TV testing using self-collected vaginal swabs to eligible pregnant women. Participants received same-day test results. Those who tested positive were given treatment. Results. Among the 225 women who were eligible and recruited, 200 (89\%) agreed to participate. The median age of our study sample was 30 years; most were unmarried (77\%), with a median gestational age of 27 weeks and a $23 \%$ HIV prevalence. All participants received their results with at least $72 \%(n=143)$ on the same day. Thirty participants $(15 \%)$ tested positive for an STI, all were treated, and $24(80 \%)$ were treated on the same day. Conclusion. The acceptability of STI testing was high, and the intervention was feasible. This study provides support for continued research into STI prevalence, cost-effectiveness, and the association of STIs with adverse maternal and infant outcomes.
\end{abstract}

\section{Introduction}

The global prevalence of curable sexually transmitted infections (STIs), including Chlamydia trachomatis, Neisseria gonorrhoeae, and Trichomonas vaginalis, remains high, and a more robust commitment to prevention, identification, and treatment is needed [1]. Untreated STIs have been associated with adverse maternal and birth outcomes [1, 2]. Recent research has identified Chlamydia trachomatis, Neisseria gonorrhoeae, and Trichomonas vaginalis as possible causes of preterm labor and birth [3-7]. Further, mother-tochild transmission of HIV may also be associated with other 
concurrent STIs [8]. One study of HIV-infected women in Tanzania found that coinfection with Neisseria gonorrhoeae was associated with a 5.5-fold increased risk of intrauterine HIV transmission [9].

Despite the adverse impact of STIs on pregnancy and birth outcomes, improvements in STI detection with molecular testing, and availability of one-dose oral treatment regimens, few countries have guidelines that recommend routine STI testing and treatment for pregnant women [1016].

In many low- and middle-income countries, including Botswana, diagnosis of STIs is primarily through a "syndromic approach." That approach utilizes an algorithm to classify symptoms and clinical signs into STI syndromes and patients are treated with standardized drug regimens [17, 18]. However, Chlamydia trachomatis, Neisseria gonorrhoeae, and Trichomonas vaginalis are often asymptomatic. Previous research has estimated that only $5-30 \%$ of women with chlamydial, gonococcal, or trichomonal infections develop symptoms [19-22]. Thus, the syndromic approach lacks sensitivity, missing a large proportion of infections, and lacks specificity, causing pregnant women to be potentially unnecessarily exposed to antibiotics $[23,24]$. Finally, as a result of the predominance of the syndromic approach, little is known about the global prevalence of Chlamydia trachomatis, Neisseria gonorrhoeae, and Trichomonas vaginalis infections among pregnant women.

From July to October 2015, we offered testing and treatment for Chlamydia trachomatis, Neisseria gonorrhoeae, and Trichomonas vaginalis in the antenatal care clinic at Princess Marina Hospital in Gaborone, Botswana. Here, we present data on the acceptability and feasibility of testing and treatment among pregnant women.

\section{Materials and Methods}

We conducted a prospective cohort study to determine STI prevalence, treatment uptake, and cure rates among pregnant women receiving outpatient antenatal care at Princess Marina Hospital in Gaborone, Botswana. We offered STI diagnosis using a GeneXpert ${ }^{\circledR}$ system (Cepheid, Sunnyvale, CA) to eligible women receiving care at the clinic during the intervention period. Women were eligible if they were 18 years or older, had a gestational age of less than 35 weeks, were mentally competent to understand the informed consent, and were willing to return to the clinic for follow-up care. The gestational age cut-off and follow-up criteria were selected to help ensure that women testing positive for an STI could return for a test of cure after four weeks and prior to delivery.

Princess Marina, located in Botswana's capital city, is the main government referral hospital for southern Botswana; approximately $85 \%$ of all births in Gaborone occur there [25]. After several weeks of shadowing the providers and integrating the study staff into the vitals room of the clinic, the intervention period began on July 9, 2015, and continued through March 2016.

2.1. Description of Intervention. At the start of each clinic day, during the general morning announcements, clinic nurses discussed the availability of STI testing for women meeting the eligibility criteria described above. The announcement included information about the importance of STI diagnosis and treatment during pregnancy. After vitals were collected and recorded by study staff, women were preliminarily screened for eligibility via review of their obstetric records. If eligible, they were provided with additional information about Chlamydia trachomatis, Neisseria gonorrhoeae, and Trichomonas vaginalis during pregnancy. Women were informed about the global frequency of those infections, their asymptomatic nature, the possible consequences to pregnant women and infants, and the recommended treatments. Women were also informed about the requirements of participation (e.g., they will be asked to provide basic demographic, behavioral, and health history information), the testing procedure via a self-collected vaginal sample, the expected 90-minute wait time, and the need for retest to evaluate the cure rate after four weeks if the results were positive. The procedure for collecting a vaginal sample was explained verbally with the help of Figure 1. Eligible women were also told that if they test positive, they would be advised to inform their partners.

Those consenting were enrolled, instructed on how to self-collect a vaginal swab specimen, and asked to provide demographic, behavioral, and health history information. We collected information on the patients' age, marital status, education level, current gestational age by last normal menstrual period (LNMP) and by ultrasound scan, birth history, HIV status, and prior syphilis diagnosis from the patient obstetric record. We also collected information about prior STI diagnoses and current symptoms that could be associated with an STI via interviews conducted by study staff in Setswana.

The specimens were tested by trained study staff for Chlamydia trachomatis, Neisseria gonorrhoeae, and Trichomonas vaginalis infections using a GeneXpert system (Cepheid, Sunnyvale, CA). Xpert provides 90-minute detection and differentiation of Chlamydia trachomatis and Neisseria gonorrhoeae and 59 minutes for Trichomonas vaginalis. Performance evaluations have found that the sensitivity for the Xpert Chlamydia trachomatis test in females using a vaginal sample is $98.7 \%$ (95\% CI: 93.1-100), Neisseria gonorrhoeae is $100.0 \%$ (95\% CI: $87.3-100$ ), and Trichomonas vaginalis is 100\% (95\% CI: 75.3-100.0) [26-28]. The specificity for the Xpert Chlamydia trachomatis test in females using a vaginal sample is $99.4 \%$ (95\% CI: 98.9-99.7), Neisseria gonorrhoeae is 99.9\% (95\% CI: 99.6-100), and Trichomonas vaginalis is $100 \%$ (95\% CI: 78.1-100.0) [26-28]. All specimens were tested onsite in the antenatal clinic vitals room.

Women were contacted about their results on the same day as testing, either in person or by telephone. If they tested positive, same-day treatment was provided before the women left the clinic. Women who tested positive were given stat doses of directly observed therapy: $1 \mathrm{~g}$ of oral azithromycin for chlamydial infection; a $250 \mathrm{mg}$ intramuscular injection of ceftriaxone in the buttocks and $1 \mathrm{~g}$ oral azithromycin for gonococcal infection (one person was not treated with azithromycin); and $2 \mathrm{~g}$ of oral metronidazole for trichomonas infection. 
(1) Open the package that contains the pink-capped Xpert swab transport reagent tube and individually wrapped collection swab, set the tube aside before beginning to collect sample, and discard the larger swab

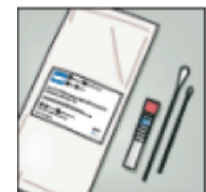

(2) Open the collection swab wrapper by peeling and open the top of the wrapper

Remove the swab, taking care not to touch the tip or lay it down

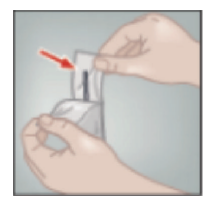

(3) Hold the swab in your hand, placing your thumb and forefinger in the middle of the swab shaft across the scoreline

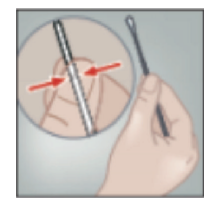

(4) Insert the swab into your vagina about two inches inside the opening of the vagina

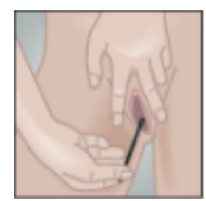

Source: Cepheid Xpert Assay training materials
(5) Gently rotate the swab for 10-30 seconds and ensure the swab touches the walls of the vagina so that moisture is absorbed by the swab Withdraw the swab

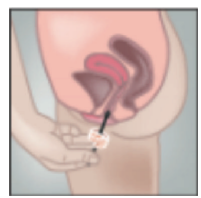

(6) Unscrew the cap from the transport tube

Immediately place the collection swab into the transport tube

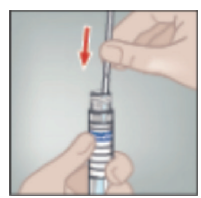

(7) Identifying the scoreline, break the swab shaft against the side of the tube and discard the top portion of the swab shaft

(8) Recap the transport tube and tighten the cap securely

Label the transport tube with the sample identification information, including date of the collection, as required

Return the tube as instructed by your doctor, nurse, or care provider
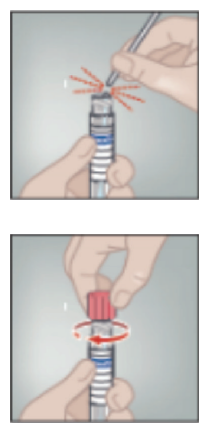

Figure 1

Those who tested positive were advised to inform their partner(s) and given the option of bringing their partner(s) with them to the antenatal clinic for treatment or giving their partner(s) a contact sheet with instructions to receive treatment at a clinic of their choosing. Additionally, for those who tested positive, a test of cure to ensure clearance of the infection was provided during their standard clinic follow-up appointment, which typically occurs after four weeks. Testing and treatment were free of charge.

The institutional review boards at the University of Botswana, the Botswana Ministry of Health, Health Research Development Committee, and Princess Marina Hospital approved the study protocol. The University of California, Los Angeles, approved analyses using deidentified data.

\section{Results and Discussion}

3.1. Acceptability of Intervention. Over the course of 12 weeks of intervention availability, we assessed 728 pregnant women for eligibility. Two hundred and seventy-three (38\%) met eligibility criteria and were offered the option of participation. The main reasons for ineligibility were having a gestational age $>34$ weeks $(n=371,82 \%)$ and not planning to receive additional care at Princess Marina $(n=62,14 \%)$.

Among the 273 eligible women, 83\% $(n=225)$ were offered participation in the intervention. We were unable to approach $100 \%$ of the eligible women largely due to study staff capacity. Occasionally, the clinic experienced a lack of running water or electricity, which temporarily caused enrollment to halt.

Among the 225 women who were eligible and offered participation, 200 (89\%) accepted. Reasons for nonacceptance of the intervention were lack of time ( $n=10,40 \%)$ and not wanting additional testing $(n=2,8 \%)$. Two (8\%) gave no reason and four (16\%) said that they would return later to test and did not. Figure 2 illustrates eligibility and acceptability over the course of the first 12 weeks of enrollment. Week 1 included two pilot days and weeks 3, 11, and 12 had low numbers of participants because they contained fewer than four days of implementation each week. The clinic volume was highest on Mondays and Tuesdays and lowest on Thursdays and Fridays. Additionally, during week 9 the clinic experienced water shortages and we were unable to enroll new patients.

Table 1 shows the characteristics of women who participated in the intervention. There were no differences in the demographic and reproductive health data collected for the women who chose not to participate (data not shown).

3.2. Feasibility. Among all women who were eligible and consented to participate in the intervention, $100 \%$ were successfully tested for Chlamydia trachomatis, Neisseria gonorrhoeae, and Trichomonas vaginalis and given their results. One sample was retested because the temperature of the Xpert machine was above the threshold. Most $(n=143,72 \%)$ were given their results in person before leaving the clinic on the 


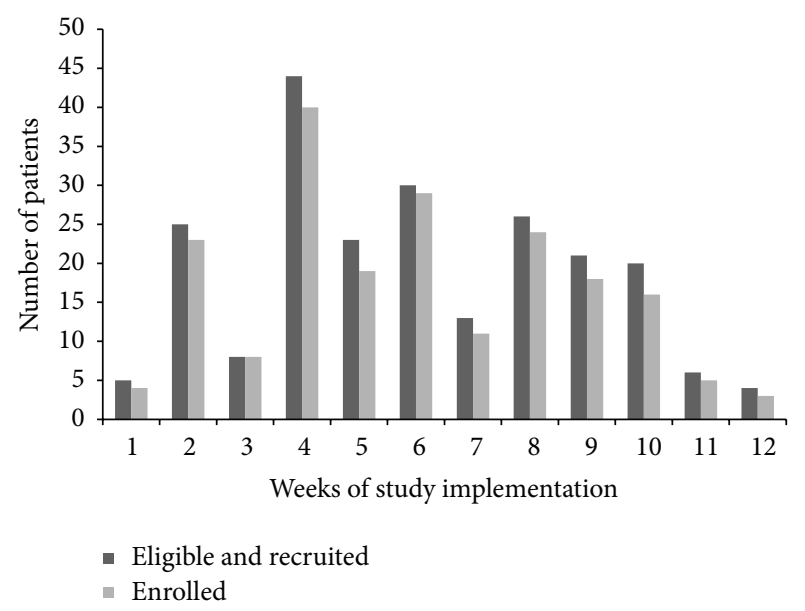

FIgURE 2: Number of patients eligible, recruited, and enrolled by week in the Chlamydia trachomatis, Neisseria gonorrhoeae, and Trichomonas vaginalis study in Gaborone, Botswana, 2015.

day of testing. However, some $(n=57,29 \%)$ were contacted by telephone, on the same day, after leaving the clinic because they had completed their clinic visit (the average wait time in clinic is 45 minutes) and could not wait for their STI results (90-minute total testing time).

The prevalence of Chlamydia trachomatis, Neisseria gonorrhoeae, or Trichomonas vaginalis in this sample was $15 \%$ $(n=30)$, with Chlamydia trachomatis at $10 \%(n=20)$, Trichomonas vaginalis at $5 \%(n=10)$, and Neisseria gonorrhoeae at $1.5 \%(n=3)$. Among the 30 women who tested positive for an STI, six (20\%) received their results over the phone and needed to return to the clinic for treatment. All women who tested positive for an STI (100\%) were successfully treated, most immediately $(80 \%)$.

\section{Discussion}

We implemented an STI testing intervention for pregnant women in the antenatal clinic of Princess Marina Hospital in Gaborone, Botswana. We found that acceptability of the intervention was high, and, as measured by the large proportion of patients who received their results on the same day as testing and the number of positives that were treated, the intervention was also feasible. All enrolled women were able to provide an adequate, self-collected vaginal sample and receive results either in person or by phone.

The results from this study are comparable to other acceptability and feasibility studies, including a recent intervention in Peru where uptake of self-collected vaginal swabs for Chlamydia trachomatis testing among pregnant women was $94 \%(600 / 640)$ [29]. Further, one study from two family planning clinics in France found the rate of Xpert assay success to be $98.3 \%$ on the first attempt using endocervical swab specimens, and the mean time of results was 2.5 hours (range: $1-5$ hours) [30].

There are limitations to our study. Women were recruited from a single site, Princess Marina Hospital antenatal clinic, which provides routine antenatal care and also serves as a
TABLE 1: Characteristics of study participants enrolled in the Chlamydia trachomatis, Neisseria gonorrhoeae, and Trichomonas vaginalis study in Gaborone, Botswana, $2015(n=200)$.

\begin{tabular}{|c|c|c|}
\hline & $n$ & $\%$ \\
\hline Total & 200 & 100 \\
\hline Age, median (IQR) & 30 & $(26-35)$ \\
\hline 18-24 years & 36 & 18 \\
\hline $25-29$ years & 58 & 29 \\
\hline $30-34$ years & 58 & 29 \\
\hline$>35$ years & 48 & 24 \\
\hline \multicolumn{3}{|l|}{ Marital status } \\
\hline Single & 152 & 76 \\
\hline Married & 46 & 23 \\
\hline Widowed & 0 & 0 \\
\hline Divorced & 0 & 0 \\
\hline Missing & 2 & 1 \\
\hline \multicolumn{3}{|l|}{ Education } \\
\hline None/primary & 5 & 3 \\
\hline Junior secondary & 52 & 26 \\
\hline Senior secondary & 65 & 33 \\
\hline Tertiary & 74 & 37 \\
\hline Missing & 4 & 2 \\
\hline Gestational age weeks (LNMP), median (range) & 27 & $(5-35)$ \\
\hline \multicolumn{3}{|l|}{ HIV status } \\
\hline Positive & 45 & 23 \\
\hline Negative & 153 & 77 \\
\hline Unknown & 2 & 1 \\
\hline Prior pregnancies, median (range) & 2 & $(1-11)$ \\
\hline Previous births, median (range) & 1 & $(0-7)$ \\
\hline \multicolumn{3}{|l|}{ Prior STI diagnosis during this pregnancy } \\
\hline No & 163 & 82 \\
\hline Yes & 27 & 14 \\
\hline Unknown & 6 & 3 \\
\hline Missing & 4 & 2 \\
\hline Positive for an STI & 30 & 15 \\
\hline Positive for Chlamydia trachomatis & 20 & 10 \\
\hline Positive for Neisseria gonorrhoeae & 3 & 1.5 \\
\hline Positive for Trichomonas vaginalis & 10 & 5 \\
\hline Dual STIs & 3 & 1.5 \\
\hline Triple STIs & 0 & 0 \\
\hline
\end{tabular}

Notes: LNMP stands for last normal menstrual period; IQR stands for interquartile range. Percentages may not add up to 100 due to rounding.

referral clinic for women with high-risk pregnancies. While nearly a quarter of our sample reported that their appointment was a routine check-up, the remainder were referred for high-risk conditions. The most common referral condition was high blood pressure. Thus, the results are not necessarily generalizable to other antenatal settings, including local clinics. Further, because the Princess Marina antenatal clinic is a referral center, most women had a gestational age greater than 34 weeks and were ineligible for our study. In order to increase efficiency, future studies should consider expanding 
enrollment to antenatal clinics attended by women earlier in their pregnancies.

Additionally, the small sample size included in this study is not powered to detect precise estimates of Chlamydia trachomatis, Neisseria gonorrhoeae, or Trichomonas vaginalis. However, it is encouraging to note that the prevalences of Chlamydia trachomatis and Neisseria gonorrhoeae in our sample are similar to those identified by Romoren et al., who found in a study of 703 antenatal care attendees in Gaborone, Botswana, between October 2000 and February 2001, that $8 \%$ and $3 \%$ were positive for Chlamydia trachomatis and Neisseria gonorrhoeae, respectively [31,32]. Further, the HIV prevalence of $23 \%$ in our sample is similar to a 2013 national survey of antenatal care attendees, which found a $20.5 \%$ prevalence of HIV among 143,037 women tested during an antenatal care visit [33]. Although our population is not representative of all pregnant women in Botswana, the results of this intervention provide evidence of uptake and feasibility using a busy, high-volume antenatal clinic in Gaborone.

Further, the intervention was run by a team of trained, dedicated study staff and was not fully integrated into the clinic using clinic staff. The study team assisted with vitals collection, providing informed consent, explaining the sample collection procedure, interviewing patients in Setswana, performing sample processing, running the GeneXpert system, and calling over one-quarter of the participants to give them their results. Calling patients with results and ensuring that some return for treatment were time-consuming. In order to facilitate same-day results and treatment, it may be necessary to decrease the already rapid testing period of 90 minutes. Further, it is clear that busy clinics would require staff dedicated to STI testing in order to ensure sustainability.

Despite those limitations, it is important to note that our intervention was able to identify and treat infections that likely would have been missed using the syndromic approach. For example, Romoren et al. estimated that the syndromic approach's sensitivity is only $49 \%$ for identifying Chlamydia trachomatis and the probability that these diagnosed women are prescribed treatment for Chlamydia trachomatis in Botswana is $85 \%[23,34]$. Thus, testing and treating STIs in pregnant women will identify more cases; however, more evidence is needed to demonstrate that routine STI testing during pregnancy is cost-effective.

\section{Conclusion}

We implemented an STI test and treat intervention for Chlamydia trachomatis, Neisseria gonorrhoeae, and Trichomonas vaginalis in the antenatal care clinic at Princess Marina Hospital in Gaborone. The acceptability of the STI testing was high, and the intervention also proved feasible. This study provides support for continued research into STI prevalence and correlates, partner notification and treatment, cost-effectiveness, and the association of STIs with adverse maternal and infant outcomes. Finally, the results may inform policy, guidelines, and decisions to make STI testing routinely available to pregnant women in Botswana and other low- and middle-income countries.

\section{Conflict of Interests}

Cepheid donated the testing reagents used in this study. The authors declare that there is no conflict of interests regarding the publication of this paper.

\section{Acknowledgments}

The authors thank Mma Kedikilwe and her nursing team for their support and contribution to the study. Also, this intervention could not have been implemented without the assistance of Ontiretsi Sickboy, Dr. Elizabeth Williams, and Sofia Duque. Adriane Wynn is a doctoral student supported by a UCLA Center for the Study of Women Spring 2015 Travel Grant, the Center for HIV Identification, Prevention, and Treatment Services (CHIPTS) NIMH Grant MH58107, and the Klausner Research and Training Fund. Ogechukwu Agatha Offorjebe and Kaitlin Arena were supported by the David Geffen School of Medicine's Dean's Office and the UCLA Center for World Health. Finally, they thank the patients for their participation.

\section{References}

[1] World Health Organization, Global Incidence and Prevalence of Selected Curable Sexually Transmitted Infections-2008, World Health Organization, Geneva, Switzerland, 2012.

[2] Institute of Medicine, Preterm Birth: Causes, Consequences, and Prevention, Committee on Understanding Premature Birth and Assuring Healthy Outcomes, 2007.

[3] M. F. Silveira, K. G. Ghanem, E. J. Erbelding et al., "Chlamydia trachomatis infection during pregnancy and the risk of preterm birth: a case-control study," International Journal of STD and AIDS, vol. 20, no. 7, pp. 465-469, 2009.

[4] P. Claman, B. Toye, R. W. Peeling, P. Jessamine, and J. Belcher, "Serologic evidence of Chlamydia trachomatis infection and risk of preterm birth," Canadian Medical Association Journal, vol. 153, no. 3, pp. 259-262, 1995.

[5] M. Gencay, M. Koskiniemi, P. Ämmälä et al., "Chlamydia trachomatis seropositivity is associated both with stillbirth and preterm delivery," APMIS, vol. 108, no. 9, pp. 584-588, 2000.

[6] M. F. Cotch, J. G. Pastorek II, R. P. Nugent et al., "Trichomonas vaginalis associated with low birth weight and preterm delivery," Sexually Transmitted Diseases, vol. 24, no. 6, pp. 353-360, 1997.

[7] G. I. J. G. Rours, R. R. de Krijger, A. Ott et al., "Chlamydia trachomatis and placental inflammation in early preterm delivery," European Journal of Epidemiology, vol. 26, no. 5, pp. 421-428, 2011.

[8] L. F. Johnson and D. A. Lewis, "The effect of genital tract infections on HIV-1 shedding in the genital tract: a systematic review and meta-analysis," Sexually Transmitted Diseases, vol. 35, no. 11, pp. 946-959, 2008.

[9] W. Fawzi, G. Msamanga, B. Renjifo et al., "Predictors of intrauterine and intrapartum transmission of HIV-1 among Tanzanian women,” AIDS, vol. 15, no. 9, pp. 1157-1165, 2001.

[10] C. C. Bristow, "Syndromic diagnostic approaches togenitourinary tract infections," in Molecular Microbiology: Diagnostic Principles and Practice, American Society for Microbiology Press, 3rd edition, 2015. 
[11] Public Health Agency of Canada, Report on Sexually Transmitted Infections in Canada: 2010, Public Health Agency of Canada, 2012.

[12] D. S. LaMontagne, K. A. Fenton, S. Randall, S. Anderson, and P. Carter, "Establishing the National Chlamydia Screening Programme in England: results from the first full year of screening," Sexually Transmitted Infections, vol. 80, no. 5, pp. 335-341, 2004.

[13] U.S. Preventive Services Task Force, "Screening for chlamydial infection: U.S. preventive services task force recommendation statement," Annals of Internal Medicine, vol. 147, no. 2, pp. 128134, 2007.

[14] N. Low, "Publication of report on chlamydia control activities in Europe," Euro Surveillance, vol. 13, no. 28, 2008.

[15] T. M. Weissenbacher, M. S. Kupka, F. Kainer, K. Friese, and I. Mylonas, "Screening for Chlamydia trachomatis in pregnancy: a retrospective analysis in a German urban area," Archives of Gynecology and Obstetrics, vol. 283, no. 6, pp. 1343-1347, 2011.

[16] K. A. Workowski and S. Berman, "Sexually transmitted diseases treatment guidelines, 2010," MMWR Recommendations and Reports, vol. 59, no. RR12, pp. 1-110, 2010.

[17] World Health Organization, Global Strategy for the Prevention and Control of Sexually Transmitted Infections: 2006-2015, 2007, http://www.who.int/hiv/pub/toolkits/stis_strategy[1]en.pdf.

[18] P. Mayaud and D. Mabey, "Approaches to the control of sexually transmitted infections in developing countries: old problems and modern challenges," Sexually Transmitted Infections, vol. 80, no. 3, pp. 174-182, 2004.

[19] Centers for Disease Control and Prevention, "TrichomoniasisCDCFact Sheet," 2015, http://www.cdc.gov/std/trichomonas/ stdfact-trichomoniasis.htm.

[20] T. A. Farley, D. A. Cohen, and W. Elkins, "Asymptomatic sexually transmitted diseases: the case for screening," Preventive Medicine, vol. 36, no. 4, pp. 502-509, 2003.

[21] E. L. Korenromp, M. K. Sudaryo, S. J. De Vlas et al., "What proportion of episodes of gonorrhoea and chlamydia becomes symptomatic?" International Journal of STD and AIDS, vol. 13, no. 2, pp. 91-101, 2002.

[22] R. Platt, P. A. Rice, and W. M. McCormack, "Risk of acquiring gonorrhea and prevalence of abnormal adnexal findings among women recently exposed to gonorrhea," The Journal of the American Medical Association, vol. 250, no. 23, pp. 3205-3209, 1983.

[23] M. Romoren, M. Rahman, J. Sundby, and P. Hjortdahl, "Chlamydia and gonorrhoea in pregnancy: effectiveness of diagnosis and treatment in Botswana," Sexually Transmitted Infections, vol. 80, no. 5, pp. 395-400, 2004.

[24] M. Romoren, M. Velauthapillai, M. Rahman, J. Sundby, E. Klouman, and P. Hjortdahl, "Trichomoniasis and bacterial vaginosis in pregnancy: inadequately managed with the syndromic approach," Bulletin of the World Health Organization, vol. 85, no. 4, pp. 297-304, 2007.

[25] J. Y. Chen, A. C. Ogwu, P. Svab et al., "Antiretroviral treatment initiation among HIV-infected pregnant women with low CD4+ cell counts in Gaborone, Botswana," Journal of Acquired Immune Deficiency Syndromes, vol. 54, no. 1, pp. 102-106, 2010.

[26] C. A. Gaydos, B. Van Der Pol, M. Jett-Goheen et al., "Performance of the cepheid CT/NG Xpert rapid PCR test for detection of Chlamydia trachomatis and Neisseria gonorrhoeae," Journal of Clinical Microbiology, vol. 51, no. 6, pp. 1666-1672, 2013.
[27] C. A. Gaydos, "Review of use of a new rapid real-time PCR, the Cepheid GeneXpert ${ }^{\circledR}$ (Xpert) CT/NG assay, for Chlamydia trachomatis and Neisseria gonorrhoeae: results for patients while in a clinical setting," Expert Review of Molecular Diagnostics, vol. 14, no. 2, pp. 135-137, 2014.

[28] S. G. Badman, L. M. Causer, R. Guy et al., "Rapid laboratory assessment of a new [Gx] GeneXpert molecular point-of-care test for detection of Trichomonas vaginalis," in Proceedings of the Australasian Sexual Health Conference, Sydney, Australia, 2014.

[29] J. Cabeza, P. J. García, E. Segura et al., "Feasibility of Chlamydia trachomatis screening and treatment in pregnant women in Lima, Peru: a prospective study in two large urban hospitals," Sexually Transmitted Infections, vol. 91, pp. 7-10, 2015.

[30] N. Bourgeois-Nicolaos, F. Jaureguy, S. Pozzi-Gaudin et al., "Benefits of rapid molecular diagnosis of Chlamydia trachomatis and Neisseria gonorrhoeae infections in women attending family planning clinics," Sexually Transmitted Diseases, vol. 42, no. 11, pp. 652-653, 2015.

[31] M. Romoren, J. Sundby, M. Velauthapillai, M. Rahman, E. Klouman, and P. Hjortdahl, "Chlamydia and gonorrhoea in pregnant Batswana women: time to discard the syndromic approach?" BMC Infectious Diseases, vol. 7, article 27, 2007.

[32] R. M. Chico, P. Mayaud, C. Ariti, D. Mabey, C. Ronsmans, and D. Chandramohan, "Prevalence of malaria and sexually transmitted and reproductive tract infections in pregnancy in sub-Saharan Africa: a systematic review," The Journal of the American Medical Association, vol. 307, no. 19, pp. 2079-2086, 2012.

[33] Statistics Botswana NACA and Ministry of Health, Botswana AIDS Impact Survey 2013 (BAIS IV 2013), http://govportal .imexsystems.net/en-gb/Documents/Ministry\%20of\%20State \%20President/NACA/Botswana\%20AIDS\%20Impact\%20Survey \%20IV\%20Report.pdf.

[34] M. Romoren, F. Hussein, T. W. Steen et al., "Costs and health consequences of chlamydia management strategies among pregnant women in sub-Saharan Africa," Sexually Transmitted Infections, vol. 83, no. 7, pp. 558-566, 2007. 


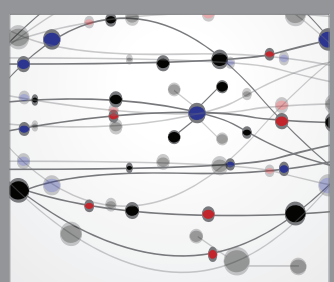

The Scientific World Journal
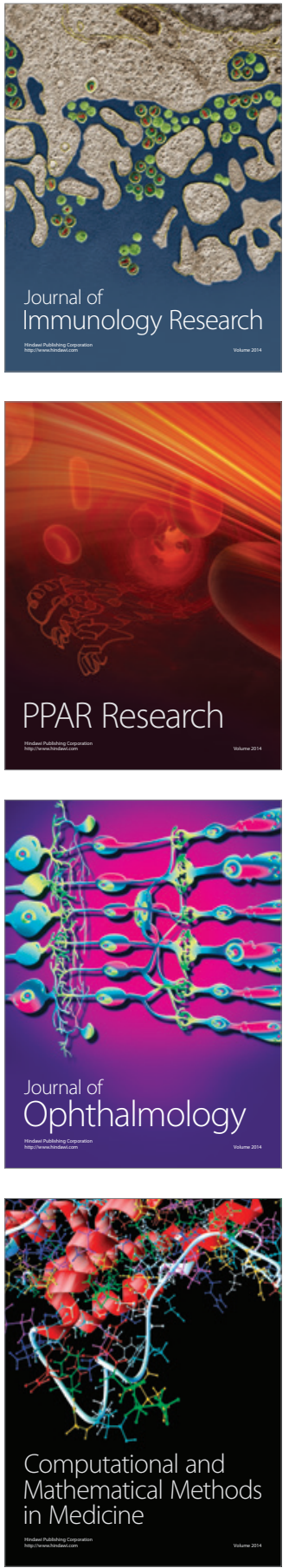

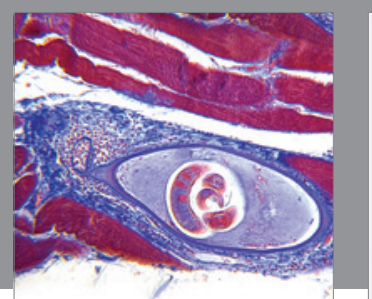

Gastroenterology Research and Practice

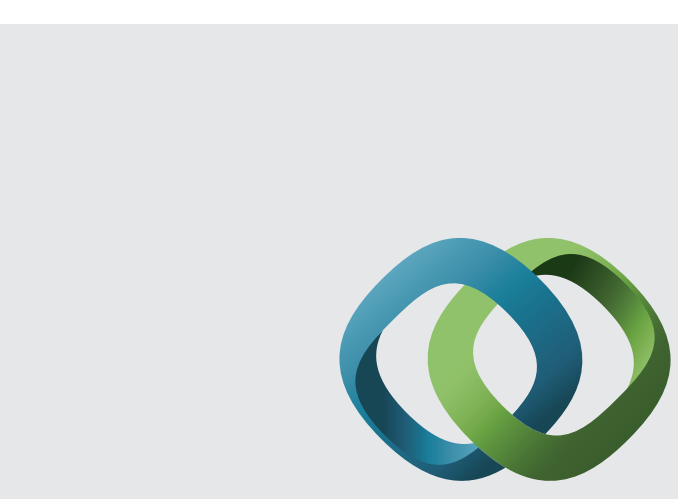

\section{Hindawi}

Submit your manuscripts at

http://www.hindawi.com
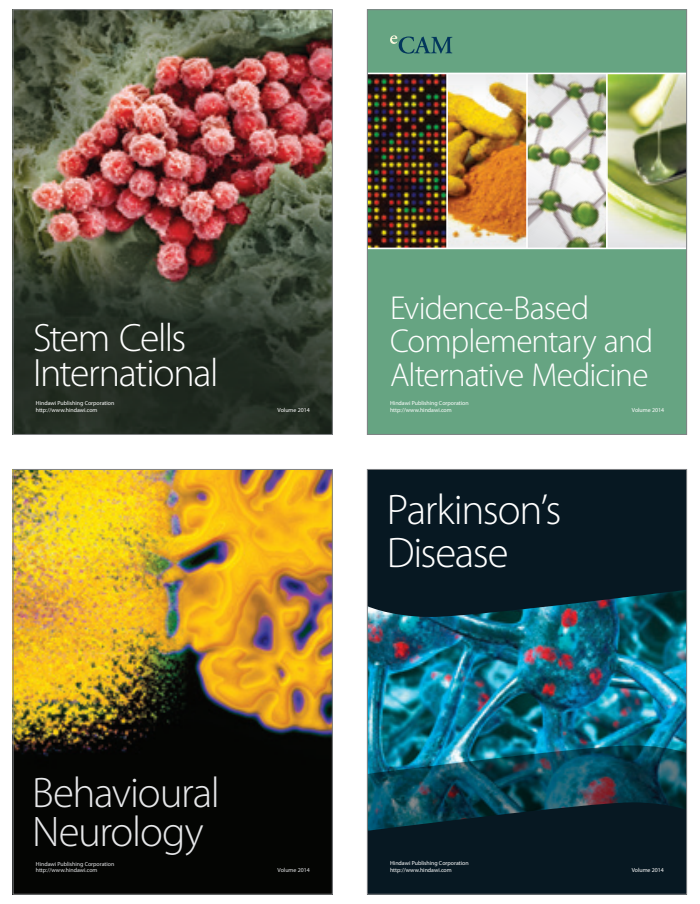
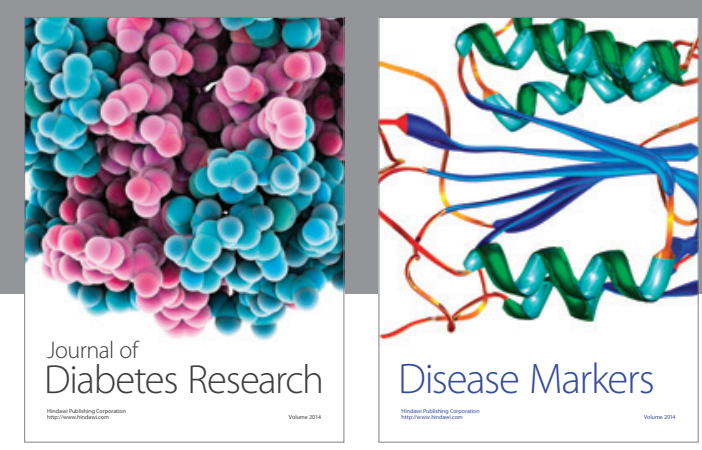

Disease Markers
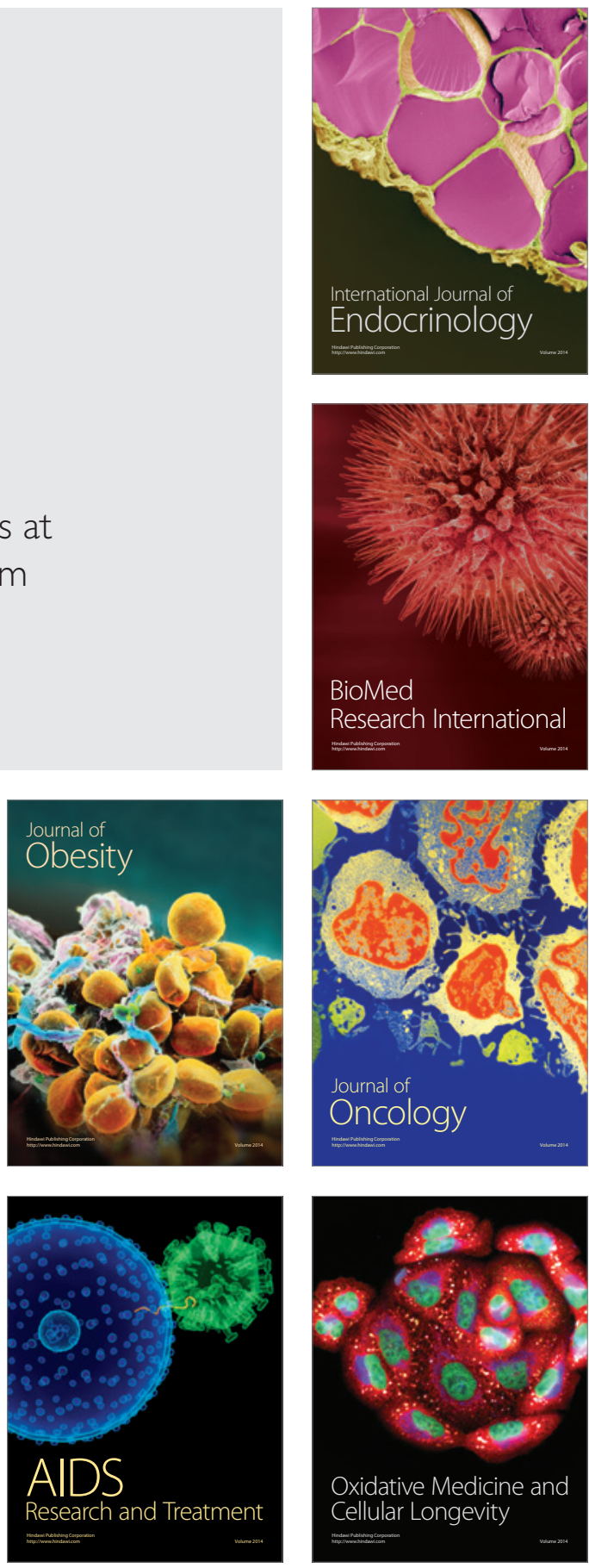